\title{
ROBUST DISSIPATIVITY FOR UNCERTAIN IMPULSIVE DYNAMICAL SYSTEMS
}

\author{
BIN LIU, XINZHI LIU, AND XIAOXIN LIAO
}

Received 5 April 2002 and in revised form 30 May 2002

We discuss the robust dissipativity with respect to the quadratic supply rate for uncertain impulsive dynamical systems. By employing the Hamilton-Jacobi inequality approach, some sufficient conditions of robust dissipativity for this kind of system are established. Finally, we specialize the obtained results to the case of uncertain linear impulsive dynamical systems.

\section{Introduction}

In many engineering problems, stability issues are often linked to the theory of dissipative systems which postulates that the energy dissipated inside a dynamical system is less than the energy supplied from an external source. In the literature of nonlinear control, dissipativity concept was initially introduced by Willems in his seminal two-part papers $[14,15]$ in terms of an inequality involving the storage function and supply rate. The extension of the work of Willems to the case of affine nonlinear systems was carried out by Hill and Moylan (see [7,8] and the references therein).

Dissipativity theory along with its connections to Lyapunov stability theory have been mainly applied to dynamical systems possessing continuous motions. However, there are many real-world systems and natural processes which display special dynamic behavior that exhibits both continuous and discrete characteristics. For instance, many evolutionary processes, particularly some biological systems such as biological neural networks and bursting rhythm models in pathology, are characterized by abrupt changes of states at certain time instants. In addition, optimal control models in economics, frequencymodulated signal processing systems, and flying object motions may also exhibit the same feature. This feature is the familiar impulsive phenomenon, and the corresponding systems are called impulsive dynamical systems (see $[1,2,9,10,11,12,17])$. Recently, researchers have also introduced and studied the stability for other discontinuous dynamical systems such as hybrid systems [18], sampled-data systems [6], and discrete-event systems [13]. For all these systems, discontinuous system motions arise naturally. More recently, Haddad et al. have developed dissipativity and exponential dissipativity concepts 
for nonlinear impulsive dynamical systems and left-continuous dynamical systems (see $[3,4,5]$ and the references therein). They have extended the notions of classical dissipativity theory using generalized storage functions and supply rates for impulsive dynamical systems and left-continuous dynamical systems.

In practice, the mathematical model used in design usually has some uncertainties. Therefore, robust control strategy becomes important. Hence, in the dissipative synthesis problems, we should consider the robustness of dissipativity in the presence of the uncertainties.

In this paper, we consider the robust dissipativity with respect to the quadratic supply rate for uncertain impulsive dynamical systems. The uncertainty is assumed to be a nonlinear function of the state that happened on the continuous part of the system, and it is bounded by a known function. Under this condition, we derive a sufficient condition under which an uncertain impulsive dynamical system is a robust dissipative one. Finally, we specialize the results to the case of uncertain linear impulsive dynamical systems.

\section{Main results}

2.1. Dissipativity for impulsive dynamical systems. We consider the impulsive dynamical system

$$
\begin{gathered}
\dot{x}(t)=f_{c}(x(t))+g_{c}(x(t)) u_{c}(t), \quad t_{k}<t \leq t_{k}, \\
\Delta x(t)=f_{d}(x(t))+g_{d}(x(t)) u_{d}(t), \quad t=t_{k}, \\
y_{c}(t)=h_{c}(x(t)), \quad t_{k}<t \leq t_{k}, \\
y_{d}(t)=h_{d}(x(t)), \quad t=t_{k},
\end{gathered}
$$

where $x\left(t_{0}\right)=x_{0}, t \geq 0, x(t) \in \mathbb{R}^{n}, \Delta x\left(t_{k}\right)=x\left(t_{k}^{+}\right)-x\left(t_{k}\right), u_{c}(t) \in \mathbb{R}^{m_{c}}, u_{d} \in \mathbb{R}^{m_{d}}, y_{c}(t) \in$ $\mathbb{R}^{l_{c}}, y_{d} \in \mathbb{R}^{l_{d}}, f_{c}: \mathbb{R}^{n} \rightarrow \mathbb{R}^{n}$ is Lipschitz continuous and satisfies $f_{c}(0)=0, g_{c}: \mathbb{R}^{n} \rightarrow \mathbb{R}^{n \times m_{c}}$, $f_{d}: \mathbb{R}^{n} \rightarrow \mathbb{R}^{n}$ is continuous and satisfies $f_{d}(0)=0, g_{d}: \mathbb{R}^{n} \rightarrow \mathbb{R}^{n \times m_{d}}, h_{c}: \mathbb{R}^{n} \rightarrow \mathbb{R}^{l_{c}}$ and satisfies $h_{c}(0)=0$, and $h_{d}: \mathbb{R}^{n} \rightarrow \mathbb{R}^{l_{d}}$ and satisfies $h_{d}(0)=0$. We will assume that $u_{c}(\cdot)$ and $u_{d}(\cdot)$ are restricted to the class of admissible inputs consisting of measurable functions $\left(u_{c}(t), u_{d}(t)\right) \in U$ for all $t \geq 0$, where $(0,0) \in U$.

Definition 2.1. A function $\left(\gamma_{c}\left(u_{c}, y_{c}\right), \gamma_{d}\left(u_{d}, y_{d}\right)\right)$, where $\gamma_{c}: \mathbb{R}^{m_{c}} \times \mathbb{R}^{l_{c}} \rightarrow \mathbb{R}$ and $\gamma_{d}: \mathbb{R}^{m_{d}} \times$ $\mathbb{R}^{l_{d}} \rightarrow \mathbb{R}$ are such that $\gamma_{c}(0,0)=0$ and $\gamma_{d}(0,0)=0$, is called a supply rate of system $(2.1)$ if $\gamma_{c}\left(u_{c}, y_{c}\right)$ is locally integrable; that is, for all input-output pairs $u_{c}(t), y_{c}(t), \gamma_{c}\left(u_{c}, y_{c}\right)$ satisfies $\int_{t}^{\hat{t}}\left|\gamma_{c}\left(u_{c}(s), y_{c}(s)\right)\right| d s<\infty$ for any $\hat{t} \geq t \geq 0$, and $\gamma_{d}\left(u_{d}, y_{d}\right)$ is locally summable. In other words, for all input-output pairs $u_{d}\left(t_{k}\right), y_{d}\left(t_{k}\right), \gamma_{d}\left(u_{d}, y_{d}\right)$ satisfies

$$
\sum_{k \in \mathbb{N}[t, \hat{t})}\left|\gamma_{d}\left(u_{d}\left(t_{k}\right), y_{d}\left(t_{k}\right)\right)\right|<\infty
$$

where $\mathbb{N}[t, \hat{t})=\left\{k: t \leq t_{k}<\hat{t}\right\}$.

Definition 2.2. An impulsive dynamical system of the form (2.1) is said to be dissipative with respect to supply rate $\left(\gamma_{c}, \gamma_{d}\right)$ if there exists a $C^{r}(r \geq 0)$ nonnegative function $V: \mathbb{R}^{n} \rightarrow \mathbb{R}$ with $V(0)=0$, called storage function, such that, for all $\left(u_{c}, u_{d}\right) \in U$, the 
following dissipation inequality holds:

$$
V(x(t)) \leq V\left(x\left(t_{0}\right)\right)+\int_{t_{0}}^{t} \gamma_{c}\left(u_{c}(s), y_{c}(s)\right) d s+\sum_{k \in \mathbb{N}[t, \hat{t})} \gamma_{d}\left(u_{d}\left(t_{k}\right), y_{d}\left(t_{k}\right)\right),
$$

where $x(t)\left(t \geq t_{0}\right)$ is a solution to $(2.1)$ with $\left(u_{c}, u_{d}\right) \in U$ and $x\left(t_{0}\right)=x_{0}$.

In [4], several basic dissipativity results for impulsive dynamical systems have been established, one of which will be introduced in following lemma.

Lemma 2.3 [4]. An impulsive dynamical system given by (2.1) is dissipative with respect to the supply rate $\left(\gamma_{c}, \gamma_{d}\right)$ if and only if there exists a $C^{0}$ nonnegative definite function $V: \mathbb{R}^{n} \rightarrow$ $\mathbb{R}$ such that, for all $k \in \mathbb{N}=\{0,1,2, \ldots\}$,

$$
\begin{aligned}
V(x(\hat{t}))-V(x(t)) & \leq \int_{t}^{\hat{t}} \gamma_{c}\left(u_{c}(s), y_{c}(s)\right) d s, \quad t_{k}<t \leq \hat{t} \leq t_{k+1}, \\
V\left(x\left(t_{k}^{+}\right)\right)-V\left(x\left(t_{k}\right)\right) & \leq \gamma_{d}\left(u_{d}\left(t_{k}\right), y_{d}\left(t_{k}\right)\right) .
\end{aligned}
$$

Remark 2.4. If in Lemma 2.3, $V(x(\cdot))$ is $C^{r}(r \geq 1)$, then, in this case, dissipativity of the impulsive dynamical system with respect to the supply rate $\left(\gamma_{c}, \gamma_{d}\right)$ is given by

$$
\begin{aligned}
\dot{V}(x(t)) & \leq \gamma_{c}\left(u_{c}(t), y_{c}(t)\right), \quad t_{k}<t \leq t_{k+1}, \\
\Delta V\left(x\left(t_{k}\right)\right) & \leq \gamma_{d}\left(u_{d}\left(t_{k}\right), y_{d}\left(t_{k}\right)\right), \quad k \in \mathbb{N},
\end{aligned}
$$

where

$$
\begin{aligned}
\Delta V\left(x\left(t_{k}\right)\right) & =V\left(x\left(t_{k}^{+}\right)\right)-V\left(x\left(t_{k}\right)\right) \\
& =V\left(x\left(t_{k}\right)+f_{d}\left(x\left(t_{k}\right)\right)+g_{d}\left(x\left(t_{k}\right)\right) u_{d}\left(t_{k}\right)\right)-V\left(x\left(t_{k}\right)\right) .
\end{aligned}
$$

2.2. Robust dissipativity for uncertain impulsive dynamical systems. The uncertain impulsive dynamical system under our consideration can be described as follows:

$$
\begin{gathered}
\dot{x}(t)=f_{c}(x(t))+\hat{f}_{c}(x(t))+g_{c}(x(t)) u_{c}(t), \quad t_{k}<t \leq t_{k}, \\
\Delta x(t)=f_{d}(x(t))+g_{d}(x(t)) u_{d}(t), \quad t=t_{k}, \\
y_{c}(t)=h_{c}(x(t)), \quad t_{k}<t \leq t_{k}, \\
y_{d}(t)=h_{d}(x(t)), \quad t=t_{k},
\end{gathered}
$$

where $\hat{f}_{c}: \mathbb{R}^{n} \rightarrow \mathbb{R}^{n}$ represents uncertainty characterized by $\hat{f}_{c}(x)=e_{c}(x) \delta_{c}(x), \hat{f}_{c}(0)=0$, the mapping $e_{c}: \mathbb{R}^{n} \rightarrow \mathbb{R}^{n \times m}$ is a known functional matrix whose entries are smooth functions of the state and $\delta_{c}: \mathbb{R}^{n} \rightarrow \mathbb{R}^{m}$ is an unknown smooth vector function belonging to the set $\Omega_{c}=\left\{\delta_{c}(x):\left\|\delta_{c}(x)\right\| \leq\left\|n_{c}(x)\right\|\right\}$, where $n_{c}: \mathbb{R}^{n} \rightarrow \mathbb{R}^{m}$ is a given continuous function with $n_{c}(0)=0$ and $\|\cdot\|$ stands for the Euclidean norm.

Definition 2.5. System (2.7) is said to be a robust dissipative system with respect to supply rate $\left(\gamma_{c}, \gamma_{d}\right)$ if, for every $\delta_{c} \in \Omega_{c}$, the system is dissipative with respect to the supply rate $\left(\gamma_{c}, \gamma_{d}\right)$. 
122 Robust dissipativity for uncertain impulsive systems

In this paper, we focus our attention on the quadratic supply rate $\left(\gamma_{c}, \gamma_{d}\right)$ as follows:

$$
\begin{aligned}
\gamma_{c}\left(u_{c}, y_{c}\right) & =\frac{1}{2}\left\{y_{c}^{T} R_{c} y_{c}+2 y_{c}^{T} S_{c} u_{c}+u_{c}^{T} Q_{c} u_{c}\right\}, \\
\gamma_{d}\left(u_{d}, y_{d}\right) & =\frac{1}{2}\left\{y_{d}^{T} R_{d} y_{d}+2 y_{d}^{T} S_{d} u_{d}+u_{d}^{T} Q_{d} u_{d}\right\},
\end{aligned}
$$

where $R_{c}, S_{c}, Q_{c}$, and $R_{d}, S_{d}, Q_{d}$ are symmetric matrices with $R_{c} \leq 0, R_{d} \leq 0$, and $Q_{c}>0$, $Q_{d}>0$.

Theorem 2.6. Suppose that there exist functions $V: \mathbb{R}^{n} \rightarrow \mathbb{R}, l_{d}: \mathbb{R}^{n} \rightarrow \mathbb{R}^{p_{d}}, w_{d}: \mathbb{R}^{n} \rightarrow$ $\mathbb{R}^{p_{d} \times m_{d}}, P_{1 u_{d}}: \mathbb{R}^{n} \rightarrow \mathbb{R}^{1 \times m_{d}}$, and $P_{2 u_{d}}: \mathbb{R}^{n} \rightarrow \mathbb{R}^{m_{d} \times m_{d}}$ with $P_{2 u_{d}}(x) \geq 0$ for all $x \in \mathbb{R}^{n}$, such that $V(\cdot)$ is $C^{1}$ and positive definite with $V(0)=0$ and that the following conditions hold:

(C1) for all $x \in \mathbb{R}^{n}, u_{d} \in \mathbb{R}^{m_{d}}$,

$$
V\left(x+f_{d}(x)+g_{d}(x) u_{d}\right)=V\left(x+f_{d}(x)\right)+P_{1 u_{d}}(x) u_{d}+u_{d}^{T} P_{2 u_{d}}(x) u_{d}
$$

(C2) there exists a positive definite function $\lambda(x)>0$ satisfying the Hamilton-Jacobi inequality, for all $t_{k}<t \leq t_{k+1}, k \in \mathbb{N}$, given by

$$
\begin{aligned}
\frac{\partial V}{\partial x}\left[f_{c}-g_{c} Q_{c}^{-1} S_{c} h_{c}\right] & +\frac{\lambda}{2} \frac{\partial V}{\partial x} e_{c} e_{c}^{T} \frac{\partial V^{T}}{\partial x}+\frac{1}{2 \lambda} n_{c}^{T} n_{c} \\
& +\frac{1}{2} \frac{\partial V}{\partial x} g_{c} Q_{c}^{-1} g_{c}^{T} \frac{\partial V^{T}}{\partial x}+\frac{1}{2} h_{c}^{T}\left(S_{c} Q_{c}^{-1} S_{c}-R_{c}\right) h_{c} \leq 0
\end{aligned}
$$

(C3) for $t=t_{k}, k \in \mathbb{N}$,

$$
\begin{gathered}
V\left(x+f_{d}(x)\right)-V(x)-\frac{1}{2} h_{d}^{T}(x) R_{d} h_{d}(x)+l_{d}^{T}(x) l_{d}(x)=0, \\
\frac{1}{2} P_{1 u_{d}}(x)-\frac{1}{2} h_{d}^{T}(x) S_{d}+l_{d}^{T}(x) w_{d}(x)=0, \\
\frac{1}{2} Q_{d}-P_{2 u_{d}}(x)-w_{d}^{T}(x) w_{d}(x)=0 .
\end{gathered}
$$

Then the uncertain impulsive dynamical system given by (2.7) is a robust dissipative system with respect to the quadratic supply rate $\left(\gamma_{c}, \gamma_{d}\right)$ given by (2.8) and (2.9).

In order to prove Theorem 2.6, we first prove the following lemmas.

Lemma 2.7. If there exists a $C^{1}$ positive definite $V(x)$, with $V(0)=0$, which satisfies the Hamilton-Jacobi inequality

$$
\frac{\partial V}{\partial x} f_{c}(x)+\frac{1}{2 \gamma^{2}} \frac{\partial V}{\partial x} g_{c}(x) g_{c}^{T}(x) \frac{\partial V^{T}}{\partial x}+\frac{1}{2} h_{c}^{T}(x) h_{c}(x) \leq 0, \quad \gamma>0,
$$

then $V(x)$ must satisfy the following dissipation inequality

$$
\frac{\partial V}{\partial x} f_{c}(x)+\frac{\partial V}{\partial x} g_{c}(x) u_{c} \leq \frac{1}{2}\left\{\gamma^{2}\left\|u_{c}\right\|^{2}-\left\|y_{c}\right\|^{2}\right\}
$$


Proof. From (2.13), we get

$$
\begin{aligned}
\frac{\partial V}{\partial x} f_{c}(x) & +\frac{\partial V}{\partial x} g_{c}(x) u_{c} \\
\leq & -\frac{1}{2} h_{c}^{T}(x) h_{c}(x)-\frac{1}{2 \gamma^{2}} \frac{\partial V}{\partial x} g_{c}(x) g_{c}^{T}(x) \frac{\partial V^{T}}{\partial x} \\
& +\frac{\partial V}{\partial x} g_{c}(x) u_{c}-\frac{1}{2} \gamma^{2} u_{c}^{T} u_{c}+\frac{1}{2} \gamma^{2} u_{c}^{T} u_{c} \\
= & \frac{1}{2}\left\{\gamma^{2}\left\|u_{c}\right\|^{2}-\left\|y_{c}\right\|^{2}\right\}-\frac{\gamma^{2}}{2}\left\|u_{c}-\frac{1}{\gamma^{2}} g_{c}^{T}(x) \frac{\partial V^{T}}{\partial x}\right\|^{2} \\
\leq & \frac{1}{2}\left\{\gamma^{2}\left\|u_{c}\right\|^{2}-\left\|y_{c}\right\|^{2}\right\} .
\end{aligned}
$$

Hence, inequality (2.14) follows.

Lemma 2.8. For every $\delta_{c} \in \Omega_{c}$ and any positive definite function $\lambda(x)>0$, the following inequality holds

$$
\frac{\partial V}{\partial x} e_{c}(x) \delta_{c}(x) \leq \frac{\lambda(x)}{2} \frac{\partial V}{\partial x} e_{c}(x) e_{c}^{T}(x) \frac{\partial V^{T}}{\partial x}+\frac{1}{2 \lambda(x)} n_{c}^{T}(x) n_{c}(x)
$$

Proof. Since $\left\|\delta_{c}(x)\right\| \leq\left\|n_{c}(x)\right\|$, so for any positive definite function $\lambda(x)>0$, we get

$$
\begin{aligned}
\frac{\partial V}{\partial x} e_{c}(x) \delta_{c}(x) & \leq\left\|\frac{\partial V}{\partial x} e_{c}(x) \delta_{c}(x)\right\| \leq\left\|\frac{\partial V}{\partial x} e_{c}(x)\right\| \cdot\left\|\delta_{c}(x)\right\| \\
& \leq \frac{\lambda(x)}{2}\left\|\frac{\partial V}{\partial x} e_{c}(x)\right\|^{2}+\frac{1}{2 \lambda(x)}\left\|\delta_{c}(x)\right\|^{2} \\
& \leq \frac{\lambda(x)}{2}\left\|\frac{\partial V}{\partial x} e_{c}(x)\right\|^{2}+\frac{1}{2 \lambda(x)}\left\|n_{c}(x)\right\|^{2} \\
& =\frac{\lambda(x)}{2} \frac{\partial V}{\partial x} e_{c}(x) e_{c}^{T}(x) \frac{\partial V^{T}}{\partial x}+\frac{1}{2 \lambda(x)} n_{c}^{T}(x) n_{c}(x)
\end{aligned}
$$

Thus, inequality (2.16) holds.

Proof of Theorem 2.6. First, we will show $\dot{V}(x) \leq r_{c}\left(u_{c}, y_{c}\right)$, for all $t_{k}<t \leq t_{k+1}, k \in \mathbb{N}$.

Since $Q_{c}>0, R_{c} \leq 0$, we can find nonsingular matrices $D$ and $E$ such that $Q_{c}=E^{T} E$, $S_{c} Q_{c}^{-1} S_{c}-R_{c}=D^{T} D$, and such that the transformation

$$
\left(\begin{array}{l}
\tilde{u}_{c} \\
\tilde{y}_{c}
\end{array}\right)=\left(\begin{array}{cc}
E & E Q_{c}^{-1} S_{c} \\
0 & D
\end{array}\right)\left(\begin{array}{l}
u_{c} \\
y_{c}
\end{array}\right)
$$

is nonsingular.

Let $\tilde{f}_{c}(x)=f_{c}(x)-g_{c}(x) Q_{c}^{-1} S_{c} h_{c}(x), \tilde{g}_{c}(x)=g_{c}(x) E^{-1}$, and $\tilde{h}_{c}(x)=D h_{c}(x)$, then system (2.7), with respect to the supply rate $\left(r_{c}, r_{d}\right)$, given by (2.8) and (2.9), changes into 
124 Robust dissipativity for uncertain impulsive systems

the form

$$
\begin{gathered}
\dot{x}(t)=\tilde{f}_{c}(x(t))+e_{c}(x) \delta_{c}(x)+\tilde{g}_{c}(x(t)) \tilde{u}_{c}(t), \quad t_{k}<t \leq t_{k}, \\
\Delta x(t)=f_{d}(x(t))+g_{d}(x(t)) u_{d}(t), \quad t=t_{k}, \\
\tilde{y}_{c}(t)=\tilde{h}_{c}(x(t)), \quad t_{k}<t \leq t_{k}, \\
y_{d}(t)=h_{d}(x(t)), \quad t=t_{k},
\end{gathered}
$$

with respect to the supply rate $\left(r_{c}, r_{d}\right)$; here,

$$
\tilde{r}_{c}\left(\tilde{u}_{c}, \tilde{y}_{c}\right)=\frac{1}{2}\left\{\left\|\tilde{u}_{c}\right\|^{2}-\left\|\tilde{y}_{c}\right\|^{2}\right\} .
$$

Hence, system (2.7) is robust dissipative with respect to the supply rate $\left(r_{c}, r_{d}\right)$, given by (2.8) and (2.9), if and only if system (2.19) is robust dissipative with respect to the supply rate $\left(\tilde{r}_{c}, r_{d}\right)$, given by $(2.9)$ and $(2.20)$.

From (C2) and Lemma 2.8, we get

$$
\begin{aligned}
\frac{\partial V}{\partial x}\left(\tilde{f}_{c}(x)\right. & \left.+e_{c}(x) \delta_{c}(x)\right)+\frac{1}{2} \frac{\partial V}{\partial x} \tilde{g}_{c}(x) \tilde{g}_{c}^{T}(x) \frac{\partial V^{T}}{\partial x}+\frac{1}{2} \tilde{h}_{c}^{T}(x) \tilde{h}_{c}(x) \\
\leq & \frac{\partial V}{\partial x} \tilde{f}_{c}(x)+\frac{\lambda(x)}{2} \frac{\partial V}{\partial x} e_{c}(x) e_{c}^{T}(x) \frac{\partial V^{T}}{\partial x}+\frac{1}{2 \lambda(x)} n_{c}^{T}(x) n_{c}(x) \\
& +\frac{1}{2} \frac{\partial V}{\partial x} \tilde{g}_{c}(x) \tilde{g}_{c}^{T}(x) \frac{\partial V^{T}}{\partial x}+\frac{1}{2} \tilde{h}_{c}^{T}(x) \tilde{h}_{c}(x) \\
= & \frac{\partial V}{\partial x}\left\{f_{c}-g_{c} Q_{c}^{-1} S_{c} h_{c}\right\}+\frac{\lambda}{2} \frac{\partial V}{\partial x} e_{c} e_{c}^{T} \frac{\partial V^{T}}{\partial x}+\frac{1}{2 \lambda} n_{c}^{T} n_{c} \\
& +\frac{1}{2} \frac{\partial V}{\partial x} g_{c} Q_{c}^{-1} g_{c}^{T} \frac{\partial V^{T}}{\partial x}+\frac{1}{2} h_{c}^{T}\left\{S_{c} Q_{c}^{-1} S_{c}-R_{c}\right\} h_{c} \leq 0 .
\end{aligned}
$$

Hence, by Lemma 2.7, $\dot{V}(x) \leq \tilde{r}_{c}\left(\tilde{u}_{c}, \tilde{y}_{c}\right)=r_{c}\left(u_{c}, y_{c}\right)$, for all $t_{k}<t \leq t_{k+1}, k \in N$.

We will now show that $\Delta V\left(x\left(t_{k}\right)\right) \leq r_{d}\left(u_{d}\left(t_{k}\right), y_{d}\left(t_{k}\right)\right), k \in \mathbb{N}$.

Applying (C1) and (C3), we get

$$
\begin{aligned}
\Delta V(x)= & V\left(x+f_{d}(x)+g_{d}(x) u_{d}\right)-V(x) \\
= & V\left(x+f_{d}(x)\right)-V(x)+P_{1 u_{d}}(x) u_{d}+u_{d}^{T} P_{2 u_{d}}(x) u_{d} \\
= & \frac{1}{2} h_{d}^{T}(x) R_{d} h_{d}(x)-l_{d}^{T}(x) l_{d}(x)+\left\{h_{d}^{T}(x) S_{d}-2 l_{d}^{T}(x) w_{d}(x)\right\} u_{d} \\
& +u_{d}^{T}\left\{\frac{1}{2} Q_{d}-w_{d}^{T}(x) w_{d}(x)\right\} u_{d} \\
= & \frac{1}{2}\left\{h_{d}^{T}(x) R_{d} h_{d}(x)+2 h_{d}^{T}(x) S_{d} u_{d}+u_{d}^{T} Q_{d} u_{d}\right\} \\
& -\left\{l_{d}^{T}(x) l_{d}(x)+2 l_{d}^{T}(x) w_{d}(x) u_{d}+u_{d}^{T} w_{d}^{T}(x) w_{d}(x) u_{d}\right\} \\
= & r_{d}\left(u_{d}, y_{d}\right)-\left\|l_{d}(x)+w_{d}(x) u_{d}\right\|^{2} \\
\leq & r_{d}\left(u_{d}, y_{d}\right) .
\end{aligned}
$$

Hence, $\Delta V\left(x\left(t_{k}\right)\right) \leq r_{d}\left(u_{d}\left(t_{k}\right), y_{d}\left(t_{k}\right)\right)$ holds for all $k \in \mathbb{N}$. 
Thus, by Lemma 2.3, system (2.19) is robust dissipative with respect to the supply rate $\left(\tilde{r}_{c}, r_{d}\right)$, given by (2.20) and (2.9), that is, system (2.7) is robust dissipative with the supply rate $\left(r_{c}, r_{d}\right)$, given by $(2.8)$ and (2.9), as required.

In the following discussion, we will apply Theorem 2.6 to a particular class of nonexpansive impulsive dynamical systems.

Definition 2.9. A system $G$, given by (2.1) ((2.7)), is (robust) nonexpansive if $G$ is (robust) dissipative with respect to the supply rate

$$
\left(r_{c}, r_{d}\right)=\frac{1}{2}\left(\gamma_{c}^{2} u_{c}^{T} u_{c}-y_{c}^{T} y_{c}, \gamma_{d}^{2} u_{d}^{T} u_{d}-y_{d}^{T} y_{d}\right)
$$

where $\gamma_{c}>0, \gamma_{d}>0$.

Corollary 2.10. Consider system (2.7) and suppose that there exist the following functions: $V: \mathbb{R}^{n} \rightarrow \mathbb{R}, l_{d}: \mathbb{R}^{n} \rightarrow \mathbb{R}^{p_{d}}, w_{d}: \mathbb{R}^{n} \rightarrow \mathbb{R}^{p_{d} \times m_{d}}, P_{1 u_{d}}: \mathbb{R}^{n} \rightarrow \mathbb{R}^{1 \times m_{d}}$, and $P_{2 u_{d}}: \mathbb{R}^{n} \rightarrow$ $\mathbb{R}^{m_{d} \times m_{d}}$ with $P_{2 u_{d}}(x) \geq 0$ for all $x \in \mathbb{R}^{n}$, such that $V(\cdot)$ is $C^{1}$ and positive definite with $V(0)=0$ and that the following conditions hold:

$(\mathrm{C} 1)^{\prime}$ for all $x \in \mathbb{R}^{n}, u_{d} \in \mathbb{R}^{m_{d}}$,

$$
V\left(x+f_{d}(x)+g_{d}(x) u_{d}\right)=V\left(x+f_{d}(x)\right)+P_{1 u_{d}}(x) u_{d}+u_{d}^{T} P_{2 u_{d}}(x) u_{d}
$$

$(\mathrm{C} 2)^{\prime}$ there exists a positive definite function $\lambda(x)>0$ satisfying the Hamilton-Jacobi inequality, for all $t_{k}<t \leq t_{k+1}, k \in \mathbb{N}$, given by

$$
\frac{\partial V}{\partial x} f_{c}+\frac{\lambda}{2} \frac{\partial V}{\partial x} e_{c} e_{c}^{T} \frac{\partial V^{T}}{\partial x}+\frac{1}{2 \lambda} n_{c}^{T} n_{c}+\frac{1}{2 \lambda^{2}} \frac{\partial V}{\partial x} g_{c} g_{c}^{T} \frac{\partial V^{T}}{\partial x}+\frac{1}{2} h_{c}^{T} h_{c} \leq 0
$$

$(\mathrm{C} 3)^{\prime}$ for $t=t_{k}, k \in \mathbb{N}$,

$$
\begin{gathered}
V\left(x+f_{d}(x)\right)-V(x)+\frac{1}{2} h_{d}^{T}(x) h_{d}(x)+l_{d}^{T}(x) l_{d}(x)=0, \\
\frac{1}{2} P_{1 u_{d}}(x)+l_{d}^{T}(x) w_{d}(x)=0, \\
\frac{\gamma_{d}^{2}}{2} I_{m_{d}}-P_{2 u_{d}}(x)-w_{d}^{T}(x) w_{d}(x)=0 .
\end{gathered}
$$

Then the uncertain impulsive dynamical system given by (2.7) is robust nonexpansive with respect to the quadratic supply rate $\left(\gamma_{c}, \gamma_{d}\right)$, given by (2.23).

Proof. The result is a direct consequence of Theorem 2.6 with $Q_{c}=\gamma_{c}^{2} I_{m_{c}}, S_{c}=0, R_{c}=$ $-I_{l_{c}}, Q_{d}=\gamma_{d}^{2} I_{m_{d}}, S_{d}=0$, and $R_{c}=-I_{l_{d}}$, where $I$ is the identity matrix. 


\section{Specialization to uncertain linear impulsive dynamical systems}

In this section, we specialize the results of Section 2 to the case of uncertain linear impulsive dynamical systems. This kind of system can be formulated as follows:

$$
\begin{aligned}
& \dot{x}(t)=A_{c} x(t)+\hat{A}_{c} x(t)+B_{c} u_{c}(t), \quad t \neq t_{k}, \\
& \Delta x(t)=\left(A_{d}-I_{n}\right) x(t)+B_{d} u_{d}(t), \quad t=t_{k}, \\
& y_{c}(t)=C_{c} x(t), \quad t \neq t_{k}, \\
& y_{d}(t)=C_{d} x(t), \quad t=t_{k},
\end{aligned}
$$

where $x\left(t_{0}\right)=x_{0}, A_{c} \in \mathbb{R}^{n \times n}, B_{c} \in \mathbb{R}^{n \times m_{c}}, C_{c} \in \mathbb{R}^{l_{c} \times n}, A_{d} \in \mathbb{R}^{n \times n}, B_{d} \in \mathbb{R}^{n \times m_{d}}, C_{d} \in \mathbb{R}^{l_{d} \times n}$, and $\hat{A}_{c} \in \mathbb{R}^{n \times n}$ represent uncertainty. By [16, Lemma 2.1], we can conclude that $\hat{A}_{c}=$ $E_{c} \Sigma_{c} F_{c}$, where $E_{c} \in \mathbb{R}^{n \times n^{2}}$ and $F_{c} \in \mathbb{R}^{n^{2} \times n}$ are known matrices, and $\Sigma_{c} \in \mathbb{R}^{n^{2} \times n^{2}}$ is the uncertain matrix satisfying

$$
\Sigma \in \Omega_{c}=\left\{\Sigma_{c} \in \mathbb{R}^{n^{2} \times R^{2}}: \Sigma_{c}=\operatorname{diag}\left\{\varepsilon_{11}, \ldots, \varepsilon_{n n}\right\},\left|\varepsilon_{i j}\right| \leq 1 ; i, j=1,2, \ldots, n\right\} .
$$

Remark 3.1. From (3.2), it is easy to get that $\Sigma_{c}$ is characterized by $\Sigma_{c}^{T} \Sigma_{c} \leq I_{n^{2}}$.

Theorem 3.2. Suppose there exist matrices $P \in \mathbb{R}^{n \times n}, L_{d} \in \mathbb{R}^{p_{d} \times n}$, and $W_{d} \in \mathbb{R}^{p_{d} \times m_{d}}$, with $P$ positive definite, such that the following conditions are satisfied.

(a1) There exists a positive constant $\lambda$ satisfying the Riccati inequality

$$
\begin{aligned}
A_{c}^{T} P & +P A_{c}-\left(P B_{c} Q_{c}^{-1} S_{c} C_{c}+C_{c}^{T} S_{c} Q_{c}^{-1} B_{c}^{T} P\right)+2 \lambda P E_{c} E_{c}^{T} P \\
& +\frac{1}{2 \lambda} F_{c}^{T} F_{c}+2 P B_{c} Q_{c}^{-1} B_{c}^{T} P+\frac{1}{2} C_{c}^{T}\left(S_{c} Q_{c}^{-1} S_{c}-R_{c}\right) C_{c} \leq 0 .
\end{aligned}
$$

(a2) The following equations hold

$$
\begin{gathered}
A_{d}^{T} P A_{d}-P-\frac{1}{2} C_{d}^{T} R_{d} C_{d}+L_{d}^{T} L_{d}=0, \\
A_{d}^{T} P B_{d}-\frac{1}{2} C_{d}^{T} S_{d}+L_{d}^{T} W_{d}=0, \\
\frac{1}{2} Q_{d}-B_{d}^{T} P B_{d}-W_{d}^{T} W_{d}=0 .
\end{gathered}
$$

Then the uncertain linear impulsive dynamical system (3.1) is robustly dissipative with respect to the supply rate $\left(\gamma_{c}, \gamma_{d}\right)$, given by (2.8) and (2.9).

Proof. Let $V(x)=x^{T} P x$, then $V$ is $C^{1}$ and positive definite. Furthermore, let

$$
\begin{gathered}
f_{c}(x)=A_{c} x, \quad g_{c}(x)=B_{c}, \quad e_{c}(x)=E_{c}, \\
\delta_{c}(x)=\sum_{c} F_{c} x, \quad f_{d}(x)=\left(A_{d}-I_{n}\right) x, \quad g_{d}(x)=B_{d}, \\
h_{c}(x)=C_{c} x, \quad h_{d}(x)=C_{d} x, \quad n_{c}(x)=F_{c} x .
\end{gathered}
$$

This implies that $\left\|\delta_{c}(x)\right\| \leq\left\|n_{c}(x)\right\|=\sqrt{x^{T} F_{c}^{T} F_{c} x}$.

Clearly, $P_{1 u_{d}}(x)=2 x^{T} A_{d}^{T} P B_{d}$ and $P_{2 u_{d}}(x)=B_{d}^{T} P B_{d} \geq 0$.

Thus, by using Theorem 2.6, the conclusion of this theorem follows. 
Corollary 3.3. Suppose there exist matrices $P \in \mathbb{R}^{n \times n}, L_{d} \in \mathbb{R}^{p_{d} \times n}$, and $W_{d} \in \mathbb{R}^{p_{d} \times m_{d}}$, with $P$ positive definite, such that the following conditions are satisfied.

(a1)' There exists a positive constant $\lambda$ satisfying the Riccati inequality

$$
A_{c}^{T} P+P A_{c}+2 \lambda P E_{c} E_{c}^{T} P+\frac{1}{2 \lambda} F_{c}^{T} F_{c}+\frac{2}{\gamma_{c}^{2}} P B_{c} B_{c}^{T} P+\frac{1}{2} C_{c}^{T} C_{c} \leq 0 .
$$

(a2)' The following equations hold

$$
\begin{gathered}
A_{d}^{T} P A_{d}-P+\frac{1}{2} C_{d}^{T} C_{d}+L_{d}^{T} L_{d}=0, \\
A_{d}^{T} P B_{d}+L_{d}^{T} W_{d}=0, \\
\frac{\gamma_{d}^{2}}{2} I_{m_{d}}-B_{d}^{T} P B_{d}-W_{d}^{T} W_{d}=0 .
\end{gathered}
$$

Then the uncertain linear impulsive dynamical system (3.1) is robustly nonexpansive with respect to the supply rate $\left(\gamma_{c}, \gamma_{d}\right)$, given by (2.23).

Proof. The result is a direct consequence of Theorem 3.2 with $Q_{c}=\gamma_{c}^{2} I_{m_{c}}, S_{c}=0, R_{c}=$ $-I_{l_{c}}, Q_{d}=\gamma_{d}^{2} I_{m_{d}}, S_{d}=0$, and $R_{d}=-I_{l_{d}}$.

\section{Conclusions}

We have studied the robust dissipativity with respect to the quadratic supply rate for uncertain impulsive dynamical systems. By employing the Hamilton-Jacobi inequality approach, some sufficient conditions of robust dissipativity for this kind of system are established. As for the robust dissipativity with respect to the generalized supply rate for uncertain impulsive dynamical systems, we will discuss it in future papers.

\section{Acknowledgment}

This work was supported by the National Natural Science Foundation (NNSF) of China and Natural Sciences and Engineering Research Council (NSERC) of Canada.

\section{References}

[1] D. D. Bainov and P. S. Simeonov, Systems with Impulsive Effects: Stability, Theory and Applications, Ellis Horwood, England, 1989.

[2] Z. H. Guan, L. James, and G. R. Chen, On impulsive autoassociative neural networks, Neural Networks 13 (2000), no. 1, 63-69.

[3] W. M. Haddad and V.-S. Chellabonia, Dissipativity theory and stability of feedback interconnections for hybrid dynamical systems, Proceedings of the American Control Conference (Chicago, Ill), IEEE, Washington, DC, 2000, pp. 2688-2694.

[4] W. M. Haddad, V.-S. Chellabonia, and N. A. Kablar, Nonlinear impulsive dynamical systems. I. Stability and dissipativity, Proceedings of the 38th IEEE Conference on Decision and Control (Phoenix, Ariz), IEEE, Washington, DC, 1999, pp. 4404-4422.

[5] _ Nonlinear impulsive dynamical systems. II. Feedback interconnections and optimality, Proceedings of the 38th IEEE Conference on Decision and Control (Phoenix, Ariz), IEEE, Washington, DC, 1999, pp. 5225-5234. 
[6] T. Hagiwara and M. Araki, Design of a stable state feedback controller based on the multirate sampling of the plant output, IEEE Trans. Automat. Control 33 (1988), no. 9, 812-819.

[7] D. Hill and P. Moylan, The stability of nonlinear dissipative systems, IEEE Trans. Automatic Control 21 (1976), no. 5, 708-711.

[8] Dissipative dynamical systems: basic input-output and state properties, J. Franklin Inst. 309 (1980), no. 5, 327-357.

[9] V. Lakshmikantham, D. D. Baĭnov, and P. S. Simeonov, Theory of Impulsive Differential Equations, Series in Modern Applied Mathematics, vol. 6, World Scientific Publishing, New Jersey, 1989.

[10] V. Lakshmikantham and X. Liu, On quasistability for impulsive differential systems, Nonlinear Anal. 13 (1989), no. 7, 819-828.

[11] Stability Analysis in Terms of Two Measures, World Scientific Publishing, New Jersey, 1993.

[12] X. Liu, Stability results for impulsive differential systems with applications to population growth models, Dynam. Stability Systems 9 (1994), no. 2, 163-174.

[13] K. M. Passino, A. N. Michel, and P. J. Antsaklis, Lyapunov stability of a class of discrete event systems, IEEE Trans. Automat. Control 39 (1994), no. 2, 269-279.

[14] J. C. Willems, Dissipative dynamical systems. I. General theory, Arch. Rational Mech. Anal. 45 (1972), 321-351.

[15] _ Dissipative dynamical systems. II. Linear systems with quadratic supply rates, Arch. Rational Mech. Anal. 45 (1972), 352-393.

[16] F. X. Wu, Z. K. Shi, and G. Z. Dai, On robust stability of dynamic interval systems, Control Theory Appl. 18 (2001), no. 1, 113-115 (Chinese).

[17] H. Ye, A. N. Michel, and L. Hou, Stability analysis of systems with impulse effects, IEEE Trans. Automat. Control 43 (1998), no. 12, 1719-1723.

[18] Stability theory for hybrid dynamical systems, IEEE Trans. Automat. Control 43 (1998), no. 4, 461-474.

Bin Liu: Department of Control Science and Engineering, Huazhong University of Science and Technology, Wuhan 430074, China

Current address: Department of Information and Computation Science, Zhuzhou Institute of Technology, Zhuzhou 412008, China

E-mail address: oliverliu78@263.net

Xinzhi Liu: Department of Control Science and Engineering, Huazhong University of Science and Technology, Wuhan 430074, China

Current address: Department of Applied Mathematics, University of Waterloo, Waterloo, Ontario Canada N2L 3G1

E-mail address: xzliu@monotone.uwaterloo.ca

Xiaoxin Liao: Department of Control Science and Engineering, Huazhong University of Science and Technology, Wuhan 430074, China

E-mail address: xiaoxinliao@163.net 


\section{Differential Equations \& Nonlinear Mechanics}

\section{An Open Access Journal}

\section{Editor-in-Chief}

K. Vajravelu

USA

Associate Editors

N. Bellomo

Italy

J. L. Bona

USA

J. R. Cannon

USA

S.-N. Chow

USA

B. S. Dandapat

India

E. DiBenedetto

USA

R. Finn

USA

R. L. Fosdick

USA

J. Frehse

Germany

A. Friedman

USA

R. Grimshaw

UK

J. Malek

Czech Republic

J. T. Oden

USA

R. Quintanilla

Spain

K. R. Rajagopal

USA

G. Saccomandi

Italy

Y. Shibata

Japan

Ivar Stakgold

USA

Swaroop Darbha

USA

A. Tani

Japan

S. Turek

Germany

A. Wineman

USA
Website: http://www.hindawi.com/journals/denm/

Aims and Scope

Differential equations play a central role in describing natural phenomena as well as the complex processes that arise from science and technology. Differential Equations \& Nonlinear Mechanics (DENM) will provide a forum for the modeling and analysis of nonlinear phenomena. One of the principal aims of the journal is to promote cross-fertilization between the various subdisciplines of the sciences: physics, chemistry, and biology, as well as various branches of engineering and the medical sciences.

Special efforts will be made to process the papers in a speedy and fair fashion to simultaneously ensure quality and timely publication.

DENM will publish original research papers that are devoted to modeling, analysis, and computational techniques. In addition to original full-length papers, DENM will also publish authoritative and informative review articles devoted to various aspects of ordinary and partial differential equations and their applications to sciences, engineering, and medicine.

\section{Open Access Support}

The Open Access movement is a relatively recent development in academic publishing. It proposes a new business model for academic publishing that enables immediate, worldwide, barrier-free, open access to the full text of research articles for the best interests of the scientific community. All interested readers can read, download, and/or print any Open Access articles without requiring a subscription to the journal in which these articles are published.

In this Open Access model, the publication cost should be covered by the author's institution or research funds. These Open Access charges replace subscription charges and allow the publishers to give the published material away for free to all interested online visitors.

\section{Instructions for Authors}

Original articles are invited and should be submitted through the DENM manuscript tracking system at http://www.mstracking.com/ denm/. Only pdf files are accepted. If, for some reason, submission through the manuscript tracking system is not possible, you can contact denm.support@hindawi.com.

Hindawi Publishing Corporation

410 Park Avenue, 15th Floor, \#287 pmb, New York, NY 10022, USA
HINDAWI 


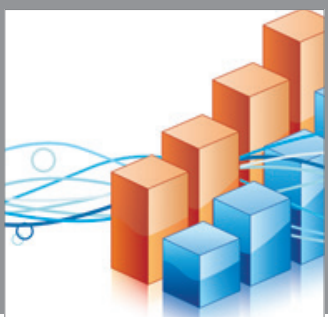

Advances in

Operations Research

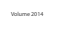

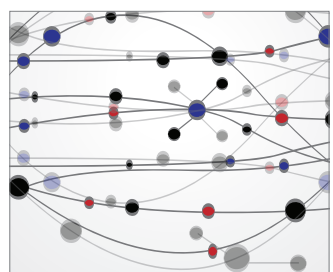

\section{The Scientific} World Journal
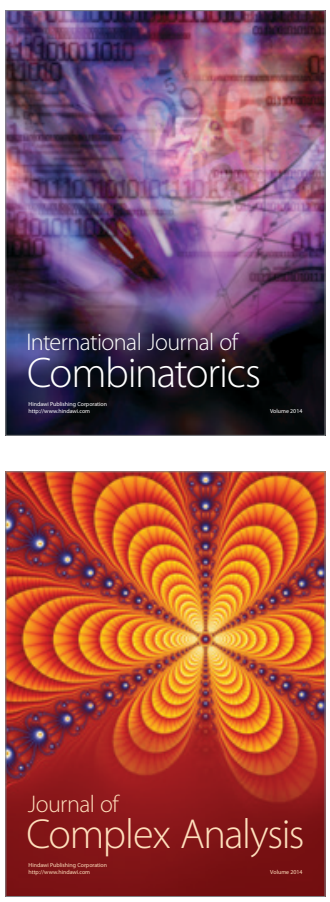

International Journal of

Mathematics and

Mathematical

Sciences
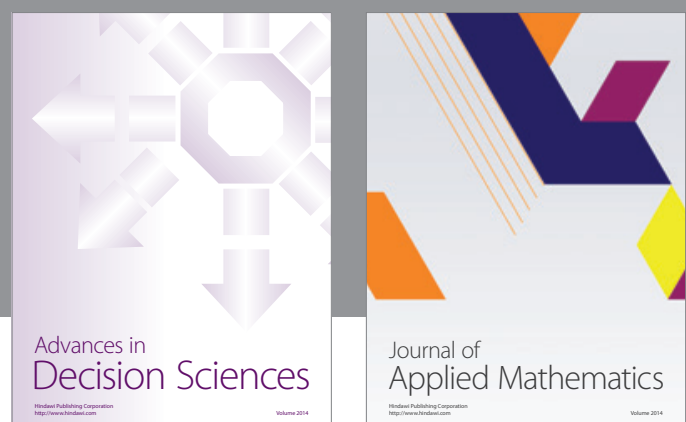

Journal of

Applied Mathematics
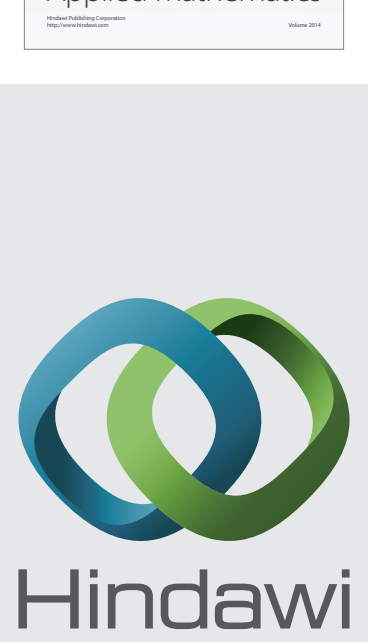

Submit your manuscripts at http://www.hindawi.com
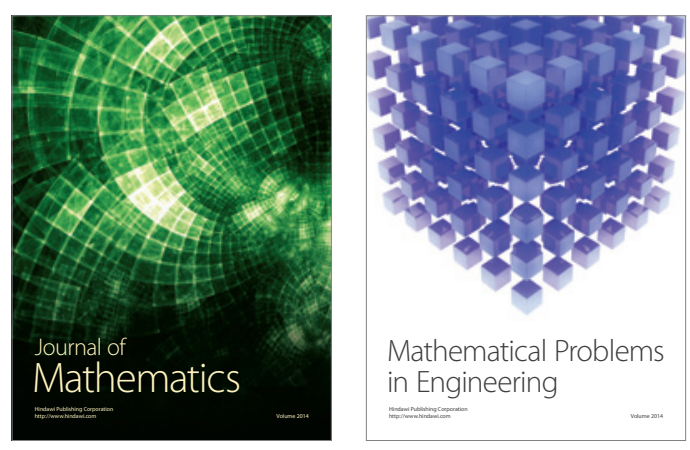

Mathematical Problems in Engineering
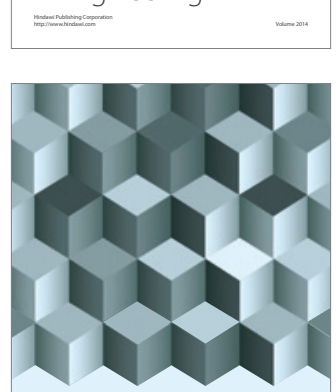

Journal of

Function Spaces
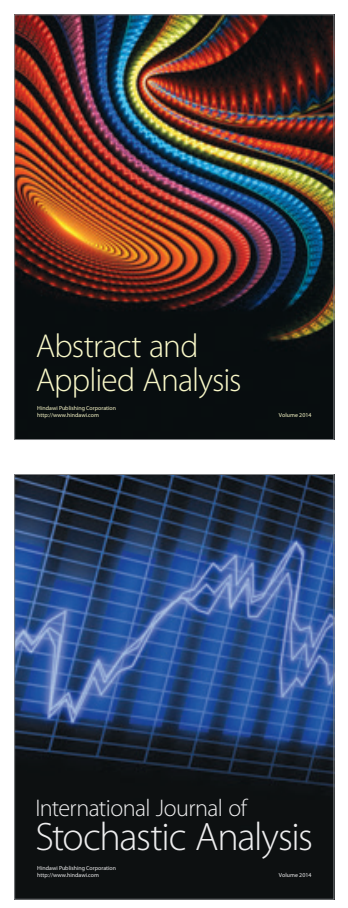

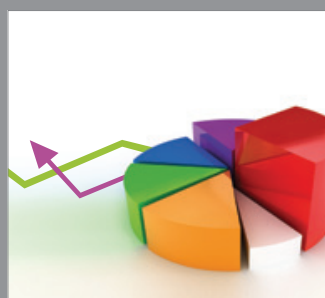

ournal of

Probability and Statistics

Promensencen
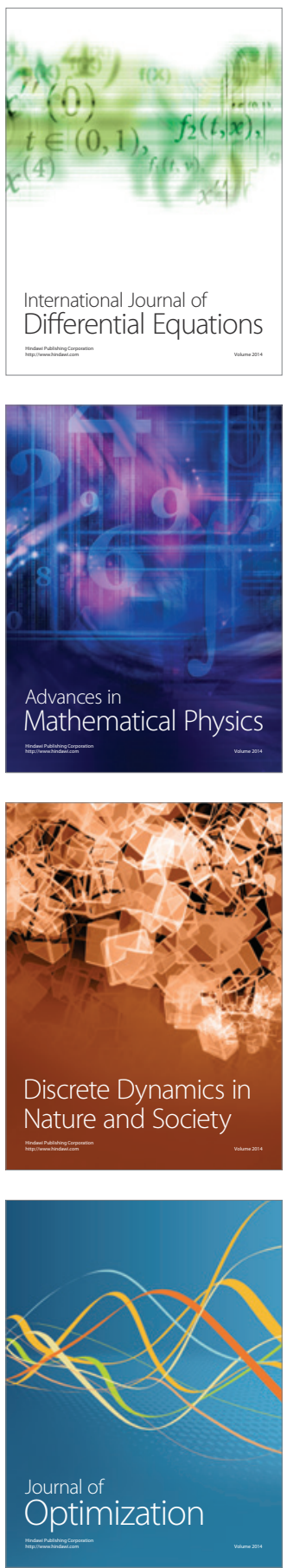